\title{
Computational modeling of the electromechanical response of a ventricular fiber affected by eccentric hypertrophy
}

\author{
Fabrizio Del Bianco ${ }^{1,2^{*}}$, Piero Colli Franzone ${ }^{3}$, Simone Scacchi ${ }^{4}$, \\ Lorenzo Fassina ${ }^{1,2}$ \\ ${ }^{1}$ Department of Electrical, Computer and Biomedical Engineering, University of \\ Pavia, Via Ferrata 1, 27100 Pavia, Italy \\ ${ }^{2}$ Centre for Health Technologies (C.H.T.), University of Pavia, Via Ferrata 1, \\ 27100 Pavia, Italy \\ ${ }^{3}$ Department of Mathematics, University of Pavia, Via Ferrata 1, 27100 Pavia, \\ Italy \\ ${ }^{4}$ Department of Mathematics, University of Milano, Via Saldini 50, 20133 \\ Milano, Italy \\ *Email address for correspondence: fabrizio.delbianco01@universitadipavia.it
}

Communicated by Simone Deparis

Received on 01 13, 2017. Accepted on 08 02, 2017.

\begin{abstract}
The aim of this work is to study the effects of eccentric hypertrophy on the electromechanics of a single myocardial ventricular fiber by means of a one-dimensional finite-element strongly-coupled model. The electrical current flow model is written in the reference configuration and it is characterized by two geometric feedbacks, i.e. the conduction and convection ones, and by the mechanoelectric feedback due to stretchactivated channels. First, the influence of such feedbacks is investigated for both a healthy and a hypertrophic fiber in case of isometric simulations. No relevant discrepancies are found when disregarding one or more feedbacks for both fibers. Then, all feedbacks are taken into account while studying the electromechanical responses of fibers. The results from isometric tests do not point out any notable difference between the healthy and hypertrophic fibers as regards the action potential duration and conduction velocity. The length-tension relationships show increased stretches and reduced peak values for tension instead. The tension-velocity relationships derived from afterloaded isotonic and quickrelease tests depict higher values of contraction velocity at smaller afterloads. Moreover, higher maximum shortenings are achieved during the isotonic contraction. In conclusion, our simulation results are innovative in predicting the electromechanical behavior of eccentric hypertrophic fibers.

Keywords: electromechanical model, mechanical feedback, eccentric hypertrophy, isometric test, afterloaded isotonic test, quick-release test

AMS subject classification: 74A05, 74A10, 74B20, 74F99, 74G15, 74L15, 74S05, $74 \mathrm{~S} 20,92 \mathrm{C} 10$
\end{abstract}




\section{F. Del Bianco et al.}

\section{Introduction}

In the whole heart, eccentric hypertrophy is an organ response to a longterm volume overload caused by physiological or pathological factors [1,2]. This yields a dilation of ventricles with a negligible wall thinning. Actually, the high diastolic wall strains developing in such an environment lead to the serial deposition of new sarcomere units inside cells without significant changes in their cross-sectional area. Eccentric hypertrophy may also be the phenotype deriving from genetic mutations that affect the correct encoding of some cytoskeletal proteins [3].

In the literature, there are a few recent studies addressing the problem of how eccentric hypertrophy can be mathematically modeled for the whole heart (e.g., [4-7]). However, they have mainly focused on the mechanical activity of hypertrophic hearts, disregarding the coupling with a model of bioelectrical activity, except from $[4,7]$. The latter ones do not take into account any mechanical feedback though.

In this paper, instead, we focus on the cardiac elementary anatomical structure, i.e. the fiber, whose contraction and relaxation processes are associated with the pumping function of the heart. In the literature, many models referring to the electromechanics of ventricular fibers have been proposed. They have been either lumped parameter models, like the ones derived from the original Hill three-element model of skeletal muscle (e.g., [8-11]), or continuous electromechanical models for one- or threedimensional fibers (e.g., $[12,13]$ ), which have only focused on free-loaded or isometric contractions.

The novelty of the present work is the development of a finite-element strongly-coupled electromechanical model that is able to investigate both the electrical and mechanical activities of eccentric hypertrophic ventricular fibers. We consider the fiber as a one-dimensional continuous strand of cardiac tissue made of electrically and mechanically coupled myocytes, through which an electrical excitation wave spreads and triggers muscle contraction.

We implement three classical in vitro protocols, i.e. the isometric, afterloaded isotonic and quick-release ones [10,14], which are known to reproduce the different types of contraction and relaxation during the four phases of a cardiac cycle: the isovolumic systole, the blood efflux, the isovolumic diastole and the diastolic filling.

The whole model consists of a zero-dimensional cardiomyocyte model of bioelectrical activity, calcium dynamics and active tension generation and a one-dimensional mechanical model of finite elasticity coupled with the Monodomain reaction-diffusion equation written in the current fiber 


\section{Electromechanical modeling of an eccentric hypertrophic fiber}

configuration and describing the electrical current flow. In a Lagrangian framework, the corresponding Monodomain equation written in the reference configuration includes two types of geometric feedback: the conduction feedback, i.e. the influence of the deformation gradient on the conductivity tensor, and the convection feedback, by introducing a dependence on the deformation rate. However, if one already wrote the convective term in the current configuration, then such a term would cancel when pulling back to the reference configuration. Although this last way to proceed is correct in case of moving particles, in this work the Monodomain model is written in the current configuration without the convective term because we follow the considerations developed in [15] and it is still not apparent from experimental data if introducing the convective term directly in the current configuration is physically valid for a moving activation wavefront among cardiomyocytes, which shift only around their reference positions. Moreover, we take into account the so-called mechanoelectric feedback represented by the influence of stretch-activated membrane channels on the ionic current [16].

In the literature, there are already several studies on the impact of mechanical feedbacks on the bioelectrical activity, starting from the first ones by Nash and Panfilov [17,18]. However, to our knowledge, no previous works have investigated the effects of all previous mechanical influences in case of both healthy and eccentric hypertrophic fibers. Therefore, this is the first problem we are going to face. Then, the remainder of this work aims at studying the electromechanical behavior of fibers contracting under isometric, afterloaded isotonic and quick-release conditions, by employing some classical measures and curves found in the literature of in vitro and in silico studies. We limit ourselves to the analysis of eccentric hypertrophy as a time response to changes in blood volumes by neglecting any genetic defect. Nevertheless, to our knowledge, for the first time, hypertrophic alterations are added both at the level of a single cardiomyocyte and at the level of the entire fiber and for both electrical and mechanical activities. Moreover, the effects of the mechanical feedback induced by a finite growth and of the changes in cardiomyocyte size on the propagation of the electrical signal are taken into account. As a remark, in the literature, there are no experimental data for eccentric hypertrophic fibers, thus our simulation results try to fill this lack of information. 


\section{F. Del Bianco et al.}

\section{Methods}

\subsection{The mechanical model}

Let us denote the material coordinates $\mathbf{X}=\left(X_{1}, X_{2}, X_{3}\right)^{T}$ of the reference cardiac domain $\widehat{H}$ and the spatial coordinates $\mathbf{x}=\left(x_{1}, x_{2}, x_{3}\right)^{T}$ of the current cardiac domain at time $t H(t)$. We denote by $\phi$ the deformation map, such that $\mathbf{x}=\phi(\mathbf{X}, t)^{T}=\phi_{t}(\mathbf{X})$, between $\widehat{H}$ and $H(t)=\phi_{t}(\widehat{H})$ and by $\mathbf{U}(\mathbf{X}, t)=\phi(\mathbf{X}, t)-\mathbf{X}$ the corresponding displacement field. In a quasistatic regime with no volume forces, we seek the displacement field $\mathbf{U}(\mathbf{X}, t)$ satisfying the equilibrium condition of the cardiac domain given by [19]

$$
\operatorname{Div}(\mathbf{F S}(\mathbf{U}, \mathbf{X}))=\mathbf{0} \quad \text { in } \widehat{H},
$$

where $\mathbf{F}=\partial \phi / \partial \mathbf{X} \equiv \partial \mathbf{x} / \partial \mathbf{X}=\mathbf{I}+\partial \mathbf{U} / \partial \mathbf{X}$ is the deformation gradient tensor, $\mathbf{S}$ is the second Piola-Kirchhoff stress tensor and $\mathbf{D i v}$ is the operator divergence defined relative to $\mathbf{X}$.

To characterize growth, we consider the multiplicative decomposition of the deformation gradient tensor $\mathbf{F}$ into an elastic part $\mathbf{F}^{\mathbf{e}}$ and a growth part $\mathbf{F}^{\mathbf{g}}$ [20] (see also, e.g., [21] for an extensive review on the state of the art of growth modeling and corresponding examples from the literature), i.e.

$$
\mathbf{F}=\mathbf{F}^{\mathrm{e}} \mathbf{F}^{\mathrm{g}}
$$

Since, during eccentric hypertrophy, $\mathbf{F}^{\mathbf{g}}$ should represent the cardiomyocytes elongation due to the serial deposition of new sarcomeres, we choose $\mathbf{F}^{\mathbf{g}}$ as in $[4,5,7]$

$$
\mathbf{F}^{\mathbf{g}}=\mathbf{I}+\left(\theta_{f}-1\right) \widehat{\mathbf{a}}_{\mathbf{f}} \otimes \widehat{\mathbf{a}}_{\mathbf{f}},
$$

where $\widehat{\mathbf{a}}_{\mathbf{f}}$ is the unit vector for the local fiber direction in $\widehat{H}$ and $\theta_{f}$ is the growth parameter along the fiber, which depends on time and space, varying typically between 1 and 2 in those computational studies dealing with eccentric hypertrophy in a ventricular model (in any case, $\theta_{f} \geq 1$ at least).

Following the active stress approach, we compute the elastic part $\mathbf{S}^{\mathbf{e}}$ of the total stress tensor $\mathbf{S}$ as

$$
\mathbf{S}^{\mathbf{e}}=\mathbf{S}^{\mathbf{e}, \mathbf{a}}+\mathbf{S}^{\mathbf{e}, \mathbf{p}}=\mathbf{F}^{\mathbf{g}} \frac{T_{a} \widehat{\mathbf{a}}_{\mathbf{f}} \otimes \widehat{\mathbf{a}}_{\mathbf{f}}}{\widehat{\mathbf{a}}_{\mathbf{f}}^{T} \mathbf{C} \widehat{\mathbf{a}}_{\mathbf{f}}}\left(\mathbf{F}^{\mathbf{g}}\right)^{T}+\frac{\partial W}{\partial \mathbf{E}^{\mathbf{e}}}-p\left(\mathbf{C}^{\mathbf{e}}\right)^{-1},
$$

where the elastic active component $\mathbf{S}^{\mathbf{e}, \mathbf{a}}$ is related to the cell active tension $T_{a}$ that is supposed to develop only along the fiber direction (see, e.g., [13, 


\section{Electromechanical modeling of an eccentric hypertrophic fiber}

$22]$ ), whilst the elastic passive component $\mathbf{S}^{\mathbf{e}, \mathbf{p}}$ models the cardiac tissue as an incompressible hyperelastic material [19]; $\mathbf{C}^{\mathbf{e}}=\left(\mathbf{F}^{\mathbf{e}}\right)^{T} \mathbf{F}^{\mathbf{e}}$ and $\mathbf{E}^{\mathbf{e}}=$ $1 / 2\left(\mathbf{C}^{\mathbf{e}}-\mathbf{I}\right)$ are the elastic parts of the right Cauchy-Green deformation tensor $\mathbf{C}$ and Lagrange-Green strain tensor $\mathbf{E}, W$ is a strain energy function and $p$ is the hydrostatic pressure.

$$
W=\frac{a}{2 b} e^{b\left(I_{1}^{e}-3\right)}+\frac{a_{f}}{2 b_{f}}\left(e^{b_{f}\left(\max \left\{I_{4 f}^{e}-1,0\right\}\right)^{2}}-1\right),
$$

where $a, b, a_{f}$ and $b_{f}$ are fixed parameters taken from [23], whilst $I_{1}^{e}=$ $\mathbf{C}^{\mathbf{e}}: \mathbf{I}$ and $I_{4 f}^{e}=\widehat{\mathbf{a}}_{\mathbf{f}}^{T} \mathbf{C}^{\mathbf{e}} \widehat{\mathbf{a}}_{\mathbf{f}}$ are the elastic invariants [4,19]. We choose a transversely isotropic constitutive law because, in this work, the fiber has equal material properties in any direction transverse to $\widehat{\mathbf{a}}_{\mathbf{f}}$. As a remark, the underlying hypothesis that a single fiber obeys the same constituive law of the whole myocardium is motivated by the fact that the former represents the elementary unit of the latter and the procedure described below to derive the one-dimensional model keeps into account the three-dimensional properties of the cardiac tissue.

Following [13,22], we assume that the cardiac domain is composed of fibers parallel to the $X_{1}$ axis, hence $\widehat{\mathbf{a}}_{\mathbf{f}}=(1,0,0)^{T}$, with no rotations occuring during deformation (i.e., from the polar decomposition, $\mathbf{F}=\mathbf{R} \mathbf{U}_{\mathbf{s}}=\mathbf{U}_{\mathbf{s}}$ with $\mathbf{R}$ a rotation tensor and $\mathbf{U}_{\mathbf{s}}$ a stretch tensor). Thus, the analytical expression of $\mathbf{S}^{\mathbf{e}, \mathbf{a}}$ simplifies into

$$
S_{M N}^{e, a}=\frac{T_{a} \theta_{f}^{2}}{C_{11}} \delta_{M 1} \delta_{N 1},
$$

where $M, N=1,2,3$ and $\delta_{i j}$ are the Kronecker symbols. Then, we suppose, like in [13], that $x_{i}(X, t)=x_{i}\left(X_{i}, t\right)$ for $i=1,2,3$. In this case, $\mathbf{F}^{\mathbf{e}}, \mathbf{C}^{\mathbf{e}}, \mathbf{E}^{\mathbf{e}}$ and $\mathbf{S}^{\mathbf{e}}$ become diagonal tensors. In particular, $\mathbf{S}^{\mathbf{e}, \mathbf{p}}$ can be written as

$$
S_{M M}^{e, p}=\frac{\partial W}{\partial E_{M M}^{e}}-\frac{p}{2 E_{M M}^{e}+1}, \quad S_{M N}^{e, p}=0 \quad \text { if } \quad M \neq N .
$$

Considering that soft tissues (like our fiber) may be characterized by relevant volume changes during growth, we set the incompressibility constraint only on the elastic part of the deformation [24], i.e. $J^{e}=\operatorname{det}\left(\mathbf{F}^{\mathbf{e}}\right)=1$, which, in terms of $\mathbf{E}^{\mathbf{e}}$, is written as

$$
\left(2 E_{11}^{e}+1\right)\left(2 E_{22}^{e}+1\right)\left(2 E_{33}^{e}+1\right)=1 .
$$

At last, we write an equation for $S_{11}^{e, p}$ as a function of $E_{11}^{e}$ following the 


\section{F. Del Bianco et al.}

procedure suggested in [13]

(9) $S_{11}^{e, p}=\frac{\partial W}{\partial E_{11}^{e}}-\frac{p}{2 E_{11}^{e}+1}=$

$$
=a e^{b\left(I_{1}^{e}-3\right)}+2 a_{f} e^{b_{f}\left(\max \left\{I_{4 f}^{e}-1,0\right\}\right)^{2}} \max \left\{I_{4 f}^{e}-1,0\right\}-\frac{p}{2 E_{11}^{e}+1} .
$$

Here, $p$ is derived from $p=\left(2 E_{22}^{e}+1\right) \partial W / \partial E_{22}^{e}=\left(2 E_{22}^{e}+1\right) a e^{b\left(I_{1}^{e}-3\right)}$ based on Equation (7), where $S_{22}^{e, p}=S_{33}^{e, p}=0$ because the traction exerted by the load acts only in the direction of the fiber; the value of $E_{22}^{e}$ is given by $E_{22}^{e}=1 /\left(2 \sqrt{2 E_{11}^{e}+1}\right)-1 / 2$ based on Equation (8), where $E_{22}^{e}=E_{33}^{e}$ because we model the fiber as a transversely isotropic structure. Since we use a one-dimensional framework in the remainder of this paper, we set

$$
\begin{gathered}
X=X_{1}, \quad x=x_{1}, \quad x(X, t): \widehat{\Omega}=[0, \widehat{L}] \rightarrow \Omega(t)=[0, L(t)], \\
F=F_{11}, \quad C=C_{11}, \quad E=E_{11}, \quad S=S_{11}^{a}+S_{11}^{p}
\end{gathered}
$$

and

$$
F^{e}=F_{11}^{e}, \quad F^{g}=F_{11}^{g}, \quad C^{e}=C_{11}^{e}, \quad E^{e}=E_{11}^{e}, \quad S^{e}=S_{11}^{e, a}+S_{11}^{e, p},
$$

where

$$
S_{11}^{e, a}=\frac{T_{a} \theta_{f}^{2}}{C_{11}}=\frac{T_{a}}{C_{11}^{e}} .
$$

After computing the elastic part $S^{e}$, the total $S$ can be derived as

$$
S=S^{e} \frac{1}{\theta_{f}},
$$

which is the simplified one-dimensional form of the corresponding threedimensional pull-back equation $\mathbf{S}=J^{g}\left(\mathbf{F}^{\mathbf{g}}\right)^{-1} \mathbf{S}^{\mathbf{e}}\left(\mathbf{F}^{\mathbf{g}}\right)^{-T}$ (with $J^{g}=$ $\left.\operatorname{det}\left(\mathbf{F}^{\mathbf{g}}\right)\right)$ from the intermediate growth configuration to the reference one.

Then, the scalar $S=S(U, X)$ enters the quasi-static equilibrium condition to seek the displacement $U(X, t)$

$$
\frac{d(F S(U, X))}{d X}=0 \quad \text { in } \quad \widehat{\Omega}
$$

with $F=1+\partial U(X, t) / \partial X$. This equation is closed by always fixing the left end, whilst the right one is fixed only under isometric conditions otherwise a load is applied to it. 


\section{Electromechanical modeling of an eccentric hypertrophic fiber}

At last, the active tension $T_{a}$ in Equation (10) is computed through the model by Land et al. [25], which can be summarized in the following way

$$
\left\{\begin{array}{l}
\frac{\partial \widehat{\mathbf{z}}}{\partial t}-\mathbf{R}_{\widehat{\mathbf{z}}}\left(\widehat{\mathbf{z}},\left[C a^{2+}\right]_{i}, \lambda^{e}, \frac{d \lambda^{e}}{d t}\right)=0 \quad \text { in } \widehat{\Omega} \times(0, T), \\
\widehat{\mathbf{z}}(\mathbf{X}, 0)=\widehat{\mathbf{z}}_{\mathbf{0}}(\mathbf{X}) \quad \text { in } \quad \widehat{\Omega}, \\
T_{a}=h\left(\widehat{\mathbf{z}}, \lambda^{e}\right) \quad \text { in } \quad \widehat{\Omega} \times(0, T),
\end{array}\right.
$$

where $\widehat{\mathbf{z}}$ is a vector of model variables in $\widehat{\Omega},\left[\mathrm{Ca}^{2+}\right]_{i}$ is the intracellular calcium concentration from the membrane model by Faber-Rudy [26] (see below) and $\lambda^{e}$ and $d \lambda^{e} / d t$ are the elastic fiber stretch and stretch rate from the mechanical model. We use $\lambda^{e}$ and $d \lambda^{e} / d t$ instead of the total ones $\lambda=\lambda^{e} \lambda^{g}=\lambda^{e} \theta_{f}$ and $d \lambda / d t$ because growth is carried out by adding new sarcomeres in cardiomyocytes rather than lengthening the preexistent ones (as observed in [7]). In particular, for a one-dimensional fiber, $\lambda^{e}$ is given by

$$
\lambda^{e}=\sqrt{C_{11}^{e}}=F_{11}^{e} .
$$

\subsection{The electrophysiological model}

Most of works on cardiac electromechanical simulations assume that the following current conservation laws, related to the intra- and extracellular current densities $\mathbf{j}_{\mathbf{i}}$ and $\mathbf{j}_{\mathbf{e}}$, hold in the current configuration $H(t)$

$$
\operatorname{div} \mathbf{j}_{\mathbf{i}}=-i_{m}+i_{\text {app }}^{i}, \quad \operatorname{div} \mathbf{j}_{\mathbf{e}}=i_{m}+i_{\text {app }}^{e},
$$

where $i_{a p p}^{i, e}$ are the intra- and extracellular applied currents and $i_{m}$ is the total transmembrane current (all of them per unit tissue volume); see [15] for a derivation of these laws and of the corresponding Bidomain model on a moving domain in case of a quasi-static regime. The transmembrane current appears as the active source or sink term depending on its sign for the two media and it establishes the coupling with a zero-dimensional membrane model. Actually, it is given by $i_{m}=i_{c}+i_{\text {ion }}$, i.e. by the sum of the capacitive membrane current $i_{c}$ and the total ionic current $i_{\text {ion }}$ flowing through membrane channels, from which we have

$$
\left\{\begin{array}{l}
i_{m}(\mathbf{x}, t)=c_{m} \frac{d v}{d t}(\mathbf{x}, t)+i_{i o n}(v, \mathbf{w}, \mathbf{c}), \\
\frac{d \mathbf{w}}{d t}-\mathbf{R}_{\mathbf{w}}(v, \mathbf{w})=0, \\
\frac{d \mathbf{c}}{d t}-\mathbf{R}_{\mathbf{c}}(v, \mathbf{w}, \mathbf{c})=0
\end{array}\right.
$$




\section{F. Del Bianco et al.}

where $c_{m}$ is the membrane capacitance, $v$ is the transmembrane potential, $\mathbf{w}$ is the vector of gating variables governing the dynamics of selective ionic channels and $\mathbf{c}$ is the vector of intracellular ionic concentrations, in particular of sodium, potassium and calcium. All variables $v, \mathbf{w}$ and $\mathbf{c}$ are defined on the current configuration $H(t)$.

We remark that what is expressed in the membrane model (16) is connected only to current flows crossing an element of the distributed cellular membrane that interconnects the intra- and extracellular media. These current flows are independent of the motion of the two media, thus all time derivatives $d(\cdot) / d t$ are partial derivatives with respect to time only. Actually, if the capacitive current, modeling the lipidic double layer of the membrane as an electric capacitor, were expressed through a total derivative of the transmembrane potential $v$, we would have

$$
i_{c}=c_{m} \frac{\partial v}{\partial t}+\operatorname{grad} v \cdot \mathbf{V},
$$

where $\mathbf{V}=\partial \phi_{t} / \partial t$ would be the velocity field of the current configuration. Then, it would follow that, for a time-constant but spatially heterogeneous transmembrane potential $v(\mathbf{x}, t)$, there could still be a capacitive current flow through the membrane due to the $\mathbf{x}$-dependence of the convective term. This would be strange and contradictory for a usual electric capacitor, for which the origin of a capacitive current is only the time variation of the transmembrane potential. A similar remark applies to the gating variables $\mathbf{w}$ related to the dynamics of channels, whose behavior is independent of the motion of the cellular membrane. Moreover, as shown in [15], the diffusive components of ionic concentrations $\mathbf{c}$ can be neglected. Therefore, even if we considered the total derivative of a generic concentration $c$

$$
\frac{D c}{D t}=\frac{\partial c}{\partial t}+\operatorname{grad} c \cdot \mathbf{V},
$$

the second term would be negligible as the diffusive flow has already been removed.

Now, it is well known that the Bidomain model in a one-dimensional fiber is equivalent to the Monodomain one (see [27,28]), which, for constant intra- and extracellular conductivity coefficients $\sigma_{f}^{i, e}$ along the fiber, is given by

$$
c_{m} \frac{\partial v}{\partial t}+i_{i o n}(v, \mathbf{w}, \mathbf{c})-\sigma_{f} \frac{\partial^{2} v}{\partial x^{2}}=i_{a p p} \quad \text { in } \quad \Omega(t)
$$

with $\sigma_{f}=\sigma_{f}^{i} \sigma_{f}^{e} /\left(\sigma_{f}^{i}+\sigma_{f}^{e}\right)$ and $i_{a p p}=\left(\sigma_{f}^{i} i_{a p p}^{i}-\sigma_{f}^{e} i_{a p p}^{e}\right)\left(\sigma_{f}^{i}+\sigma_{f}^{e}\right)$. Pulling back to the reference configuration $\widehat{\Omega}$ and considering the incompressibility 


\section{Electromechanical modeling of an eccentric hypertrophic fiber}

constraint $J^{e}=\operatorname{det}\left(\mathbf{F}^{\mathbf{e}}\right)=1$ and the relationship $J=\operatorname{det}(\mathbf{F})=J^{e} J^{g}=\theta_{f}$, the one-dimensional Monodomain model (see [15]) reads

$$
\left\{\begin{array}{l}
c_{m}\left(\frac{\partial \widehat{v}}{\partial t}-\frac{1}{F} \frac{\partial \widehat{v}}{\partial X} \frac{\partial x}{\partial t}\right)-\frac{1}{\theta_{f}} \frac{\partial}{\partial X}\left(\theta_{f} \frac{\sigma_{f}}{F^{2}} \frac{\partial \widehat{v}}{\partial X}\right)+ \\
+i_{i o n}(\widehat{v}, \widehat{\mathbf{w}}, \widehat{\mathbf{c}})+i_{S A C}\left(\widehat{v}, \widehat{\mathbf{c}}, \lambda^{e}\right)=\widehat{i}_{a p p} \quad \text { in } \quad \widehat{\Omega} \times(0, T), \\
\frac{\partial \widehat{v}}{\partial X}=0 \quad \text { on } \quad \partial \widehat{\Omega} \times(0, T), \\
\widehat{v}(X, 0)=\widehat{v}_{0}(X) \quad \text { in } \quad \widehat{\Omega}, \\
\frac{\partial \mathbf{w}}{\partial t}-\mathbf{R}_{\mathbf{w}}(v, \mathbf{w})=0 \quad \text { in } \quad \Omega(t) \times(0, T), \\
\frac{\partial \mathbf{c}}{\partial t}-\mathbf{R}_{\mathbf{c}}(v, \mathbf{w}, \mathbf{c})=0 \quad \text { in } \quad \Omega(t) \times(0, T), \\
\mathbf{w}(\mathbf{x}, 0)=\mathbf{w}_{\mathbf{0}}(\mathbf{x}), \quad \mathbf{c}(\mathbf{x}, 0)=\mathbf{c}_{\mathbf{0}}(\mathbf{x}), \quad \text { in } \quad \Omega(t),
\end{array}\right.
$$

where $\widehat{v}, \widehat{\mathbf{w}}$ and $\widehat{\mathbf{c}}$ are defined in $\widehat{\Omega}$ and $i_{\text {ion }}, i_{S A C}$ and $\widehat{i}_{\text {app }}$ are the total ionic current, the stretch-activated channels current and the applied current stimulus per unit fiber length. Since the fiber is supposed to be electrically insulated, the second equation imposes a zero Neumann boundary condition.

In our simulations, the Monodomain model is coupled with the Faber-Rudy membrane model, which gives the ionic membrane current $I_{i o n}(\widehat{v}, \widehat{\mathbf{w}}, \widehat{\mathbf{c}})$ per unit area of the membrane surface. Since we assume a constant ratio of membrane surface per volume $\chi=1000 \mathrm{~cm}^{-1}$, we have $i_{\text {ion }}=\chi I_{\text {ion }}$.

The extracellular conductivity $\sigma_{f}^{e}$ has a conservative value of $2 \mathrm{mS} / \mathrm{cm}$ (see, e.g., [28]), whilst the intracellular one $\sigma_{f}^{i}$ is computed as $[29,30]$

$$
\sigma_{f}^{i}=\frac{1}{r_{c y t}+\frac{r_{j u n c t}}{L_{c e l l}}},
$$

where $r_{c y t}$ and $r_{\text {junct }}$ are the cytoplasmic and gap junction resistivities and $L_{\text {cell }}=\theta_{f} L_{\text {cell,healthy }}$ is the cardiomyocytes length (with $L_{\text {cell,healthy }}$ the length value from the original Faber-Rudy membrane model, i.e. $0.01 \mathrm{~cm}$ ). We use the values $r_{c y t}=150 \Omega \cdot \mathrm{cm}$ and $r_{\text {junct }}=1.5 \Omega \cdot \mathrm{cm}^{2}$ from [31] to obtain a value of $3 \mathrm{mS} / \mathrm{cm}$ for $\sigma_{f}^{i}$ [28] if $\theta_{f}=1$, which, together with the conservative one chosen for $c_{m}(1 \mu \mathrm{F} / \mathrm{cm})$, ensures a conduction velocity between 0.06 and $0.07 \mathrm{~cm} / \mathrm{ms}$ in case of the healthy fiber. Moreover, the same variable $L_{\text {cell }}$ affects the geometric plasma membrane 


\section{F. Del Bianco et al.}

area $A_{\text {geo }}=2 \pi R_{\text {cell }}^{2}+2 \pi R_{\text {cell }} L_{\text {cell }}$ (where $R_{\text {cell }}$ is the cardiomyocytes radius, i.e. $0.0011 \mathrm{~cm}$ ), the capacitive one $A_{\text {cap }}=2 A_{\text {geo }}$ and the cell volume $V_{\text {cell }}=\pi R_{\text {cell }}^{2} L_{\text {cell }}$, which are used to solve the Faber-Rudy model related to the single cardiomyocyte. On the contrary, the surface-to-volume ratio $\chi$ does not depend on growth in this work.

The second term in the first equation of system (20) contains a convective term depending on the one-dimensional deformation gradient $F$ and deformation rate $\partial x / \partial t$, whereas the third term is only dependent on $F$; these two geometric feedbacks are called convection and conduction feedbacks respectively in the remainder of this work. Moreover, in the same equation, the membrane current $i_{S A C}$, depending on the elastic stretch $\lambda^{e}$, points out the addition of stretch-activated channels SACs to the original Faber-Rudy model, which define the so-called mechanoelectric feedback. In this work, we use the $I_{S A C}$ model proposed in [32] given by the sum of the following currents

$$
\begin{gathered}
I_{S A C, N a}=\bar{G}_{S A C} \gamma_{S L, S A C} \frac{E_{R}-E_{K}}{E_{N a}-E_{R}}\left(\widehat{v}-E_{N a}\right), \\
I_{S A C, K}=\bar{G}_{S A C} \gamma_{S L, S A C}\left(\widehat{v}-E_{K}\right), \\
I_{K o}=\bar{G}_{K o} \frac{\gamma_{S L, K o}}{1+e^{-\frac{10+\widehat{v}}{45}}}\left(\widehat{v}-E_{K}\right),
\end{gathered}
$$

where $\bar{G}_{S A C}=4.13 \cdot 10^{-3} \mathrm{mS} / \mathrm{cm}^{2}$ and $\bar{G}_{K o}=1.2 \cdot 10^{-2} \mathrm{mS} / \mathrm{cm}^{2}$ are two maximum channel conductances per unit area of the membrane surface, $\gamma_{S L, S A C}=10 \max \left\{\lambda^{e}-1,0\right\}$ and $\gamma_{S L, K o}=0.7+3 \max \left\{\lambda^{e}-1,0\right\}$ account for the linear dependence of currents on the elastic fiber stretch $\lambda^{e}$ and $E_{R}=$ $-10 \mathrm{mV}, E_{K}=-85 \mathrm{mV}$ and $E_{N a}=65 \mathrm{mV}$ are the reversal potentials of $I_{S A C}$, potassium and sodium. Hence, $I_{S A C}$ is activated only when $\lambda^{e}>1$ according to the experimental results reported in the literature (e.g., [16]).

During growth and all simulations, the fiber is excited for $1 \mathrm{~ms}$ by a current stimulus $\widehat{i}_{\text {app }}$ of $250 \mathrm{~mA} / \mathrm{cm}$ to its left end, which then propagates towards the right end in order to better simulate the in vivo propagation of current stimuli along fibers, unlike the usual in vitro experiments, where the entire fiber is stimulated simultaneously.

\subsection{The growth model}

Fiber growth is achieved by following to some extent the procedure suggested in [7] for five beats. No cardiac cycle phases are implemented at the level of the fiber for the sake of simplicity. For each beat, the fiber is first stretched at its right end by a load equal to $6 \mathrm{kPa}$, which should simulate the rise in ventricular end-diastolic pressure from a healthy value 


\section{Electromechanical modeling of an eccentric hypertrophic fiber}

of $2 \mathrm{kPa}$ due to an increase of atrial pressure during mitral regurgitation (a possible factor triggering eccentric hypertrophy). Then, it is stimulated in order to contract and relax without modifying its length. At last, at the end of the beat, the fiber can grow. In this work, we adopt the fixed reference configuration method to update the growth of a soft tissue (see [33] for a comparison with the updated reference configuration one). Thus, we first compute the local increments for $\theta_{f}$ between the beats $n$ and $n+1$, i.e. $\theta_{f, *}$, as

$$
\theta_{f, *}=1+k\left(\theta_{f, n}\right)\left(\overline{\lambda_{n}^{e}}-\overline{\lambda_{h}^{e}}\right) .
$$

Here, $k$ is a rate-limiting function with the expression $k=1 / \tau\left(\theta_{f}^{\max }-\right.$ $\left.\theta_{f, n}\right) /\left(\theta_{f}^{\max }-1\right)$, where $\tau=0.2$ and $\theta_{f}^{\max }=4$ (to enhance differences in local growth). The growth criterion $\overline{\lambda_{n}^{e}}-\overline{\lambda_{h}^{e}}$, instead, is given by the deviation of the local time-averaged elastic stretch during the beat $n$ from the local time-averaged homeostatic set point value dictated by a healthy simulation with a preload equal to $2 k P a$. Then, we compute the new local values for $\theta_{f}$ at the beat $n+1$, i.e. $\theta_{f, n+1}$, from the product

$$
\theta_{f, n+1}=\theta_{f, *} \theta_{f, n},
$$

where $\theta_{f, n}$ represents the cumulative growth of all previous beats up to the beat $n$.

\subsection{The discretization of the complete model}

The discretization of the complete model is performed by continuous piecewise linear finite elements in space and semi-implicit finite differences in time. The ODEs for $\mathbf{c}$ are solved by the Forward Euler method, whereas the ODEs for $\mathbf{w}$ by the Rush-Larsen scheme [34]. The active tension generation model is solved coupled with the equilibrium equation (12) using the update method [12], i.e. the variables of the active tension generation model are updated at each Newton iteration together with the resolution of the mechanical deformation. A uniform mesh of 100 linear finite elements and a constant time step $\Delta t_{e l}$ of $0.05 \mathrm{~ms}$ are used for the electrical components, whereas 50 linear finite elements and a constant time step $\Delta t_{m e c}$ of $1 \mathrm{~ms}$ (if not otherwise specified) are used for the mechanical ones.

We perform all simulations in Matlab on a one-dimensional fiber with a uniform cross-section and a reference length $\widehat{L}$ of $1 \mathrm{~cm}$ (Figure 1, panel A). Its initial length changes according to the type of fiber, i.e. it is equal to $1 \mathrm{~cm}$ for the healthy fiber and about $1.95 \mathrm{~cm}$ for the hypertrophic one, which is obtained at the end of the previously described five growth beats. 
F. Del Bianco et al.

A

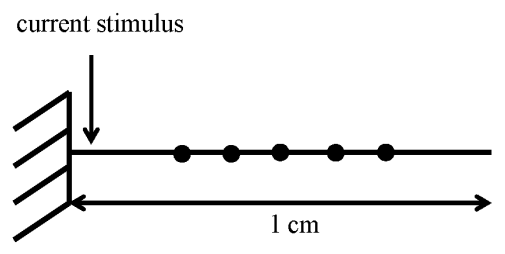

C

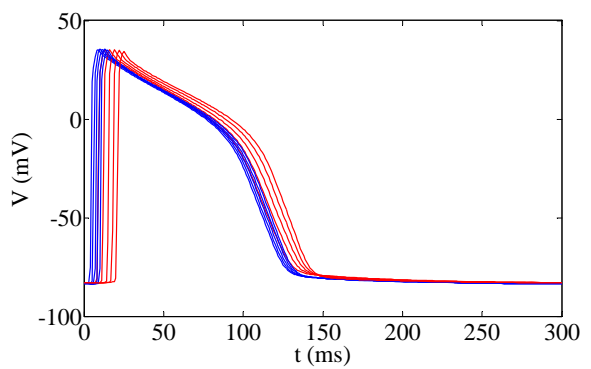

E

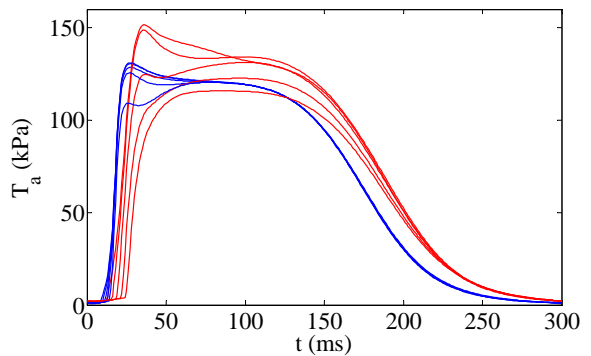

B

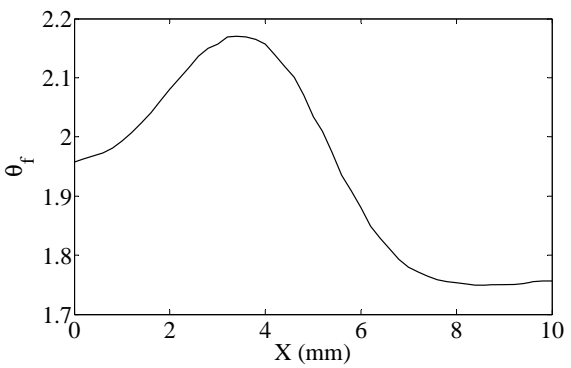

$\mathrm{D}$



$\mathrm{F}$

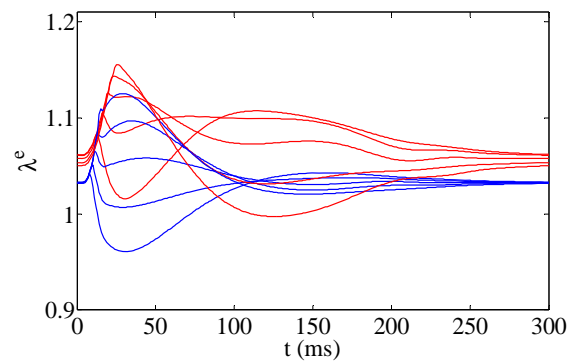

Figure 1. A) reference configuration between 0 and $1 \mathrm{~cm}$ for the healthy and hypertrophic fibers. The left end is always kept fixed, whilst the right one is fixed only during isometric contractions and relaxations, otherwise it is subjected to a load. The current stimulus is given at the left end. B) spatial distribution of the growth parameter $\theta_{f}$ shown in the reference configuration (see text for details on its derivation). C-F) time evolutions of the transmembrane potential $V(\mathrm{C})$, intracellular calcium concentration $\left[\mathrm{Ca}^{2+}\right]_{i}(\mathrm{D})$, active tension $T_{a}(\mathrm{E})$ and elastic stretch $\lambda^{e}(\mathrm{~F})$, all referring to five equally-spaced nodes (denoted by dots and selected at 3, 4, 5, 6 and $7 \mathrm{~mm}$ from the left end in A)) during an isometric simulation with a preload equal to $4 \mathrm{kPa}$ and involving the healthy (blue) or hypertrophic (red) fiber. 


\section{Electromechanical modeling of an eccentric hypertrophic fiber}

\subsection{The implemented tests}

At the beginning of all protocols, a specific preload is applied to the right end of the healthy or hypertrophic fiber to stretch it passively. Then, the fiber is fixed at the right end and five isometric beats are run to reach a steady state for that preload. The final values for nodal stretches, coordinates and all Faber-Rudy-Land model variables are saved and represent the initial conditions for all the tests made later. The stretch rate is set to zero for all nodes because the fiber stops after its stretching.

For the isometric tests, only another isometric twitch is run.

To implement the afterloaded isotonic tests, instead, we automatically fix the right end solely as long as the fiber does not contract. Thus, an initial isometric contraction phase occurs before becoming purely isotonic as soon as the developed tension (i.e. the reaction at the left end of the fiber) equals the applied load. In experimental studies, this load is the sum of the previous preload and another externally applied afterload. However, our protocol automatically sets a unique afterload, which virtually includes the preload and the afterload, by the following procedure. First, an isometric twitch is run after the preloading phase. Then, the range between the maximum and the minimum values of the developed tension is equally divided in ten parts, obtaining a constant increment step. At last, starting from the minimum value, the afterload is incremented by this constant step in an iterative way resulting in many isotonic tests that start from the same initial conditions assigned to the isometric twitch. When the afterload is too heavy, i.e. it is equal to the maximum value of the developed tension, then the last isotonic test becomes totally isometric. A simulation with a zero afterload is run for each preload too.

For the quick-release tests, which are almost similar to the afterloaded isotonic ones apart from the fact that the transition from the isometric contraction to the isotonic one is externally enforced, we let the fiber free to contract isotonically only when it develops the maximum tension due to a specific initial length. Thus, we prolong the initial isometric contraction to elicit at the same time all the subsequent isotonic contractions for different afterloads. A simulation with a zero afterload is eventually performed again.

\section{Results and discussion}

\subsection{Fiber growth}

Panel B of Figure 1 displays the resultant spatial distribution of $\theta_{f}$ at the end of the fifth growth beat on the reference configuration. The resultant non-uniformity of such a distribution mainly derives from the isometric 


\section{F. Del Bianco et al.}

beat performed before triggering growth, which has been found to enhance differences in local growth better than an isotonic one with a constant applied load, and the position of the stimulation site. Due to the fact that both fiber ends are fixed and the external stimulus is applied at the left end, a more inhomogeneous distribution of $\overline{\lambda_{n}^{e}}$ values (driving fiber growth) comes out. The nodes closer to the left end, which are the first activated ones, contract to a lesser extent, hence they show more positive $\overline{\lambda_{n}^{e}}$ values, whilst the ones closer to the right end, which are activated later, contract to a higher extent, thus they exhibit less positive $\overline{\lambda_{n}^{e}}$ values. Actually, the former nodes develop a lower value of active tension $T_{a}$ due to the lower values of $\lambda_{n}^{e}$ before their contraction compared with the latter nodes, which develop a higher value of $T_{a}$ because the $\lambda_{n}^{e}$ values are raised by the preceding contraction of the leftmost nodes.

Panels C-F represent the time evolutions of the transmembrane potential $V \equiv \widehat{v}$, intracellular calcium concentration $\left[\mathrm{Ca}^{2+}\right]_{i}$, active tension $T_{a}$ and elastic stretch $\lambda^{e}$ in five equally-spaced nodes along the healthy and hypertrophic fibers (denoted by dots and chosen at 3, 4, 5, 6 and $7 \mathrm{~mm}$ from the left end in panel A) in case of isometric simulations with a preload equal to $4 k P a$. The propagation of the electrical signal can be remarked.

\subsection{The electrical response}

The electrical responses of healthy and hypertrophic fibers are analyzed by means of isometric tests because isometric contractions and relaxations are present in case of afterloaded isotonic and quick-release tests too and isometric tests are usually found in those in silico studies aimed at investigating the eletrical behavior of contracting fibers (e.g., [8,9,11]).

The first issue we deal with is the analysis of the relevance of the mechanical feedbacks in the Monodomain model of electrophysiology (20). For both healthy and hypertrophic fibers, we run: i) a simulation with the conduction feedback (COND); ii) a simulation with the conduction and convection feedbacks $(\mathrm{COND}+\mathrm{CONV})$; iii) a simulation with the conduction and mechanoelectric feedbacks (COND+SAC); iv) a simulation with all three feedbacks (COND+CONV+SAC). For these simulations, we choose $\Delta t_{m e c}=\Delta t_{e l}$ in order to improve the accuracy of results. We report in Figures 2 and 3 the resulting spatial distributions (shown in the reference configuration) of the activation time $A T$, repolarization time $R T$, corresponding action potential duration $A P D=R T-A T$ and propagation (or conduction) velocity $v_{\text {prop }}$ for the healthy and hypertrophic fibers respectively under all simulated conditions. In this work, $A T$ is defined as the time instant when $V$ exceeds the threshold value of $-40 \mathrm{mV}, R T$ is defined as the 


\section{Electromechanical modeling of an eccentric hypertrophic fiber}

time instant when $V$ becomes less than its $90 \%$ repolarization value of -76.5 $m V$ and $v_{\text {prop }}$ is computed as the ratio of the coordinate of each node at the moment of its electrical activation to its activation time. From a visual inspection, it appears that the $A T$ of the healthy fiber is not affected at all. A maximum discrepancy of only $2 \mathrm{~ms}$ is present at the right end of the hypertrophic fiber instead. As regards both $R T$ and $A P D$, a systematic but slight difference is found along the entire healthy fiber between the COND or $\mathrm{COND}+\mathrm{CONV}$ case and the $\mathrm{COND}+\mathrm{SAC}$ or $\mathrm{COND}+\mathrm{CONV}+\mathrm{SAC}$ one. A similar difference is detected for APD only in case of the hypertrophic fiber. Moreover, $v_{\text {prop }}$ for both fibers turns out not to be strongly affected by mechanical feedbacks.
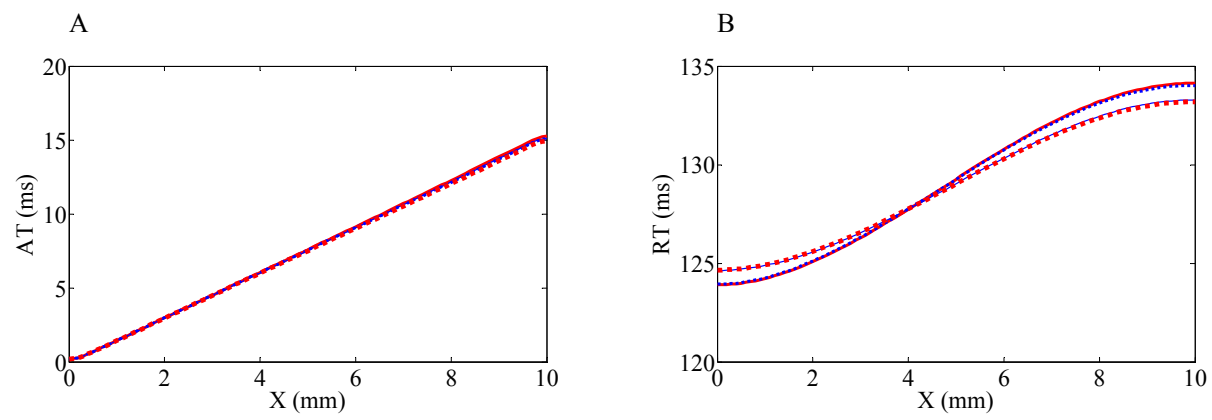

$\mathrm{C}$
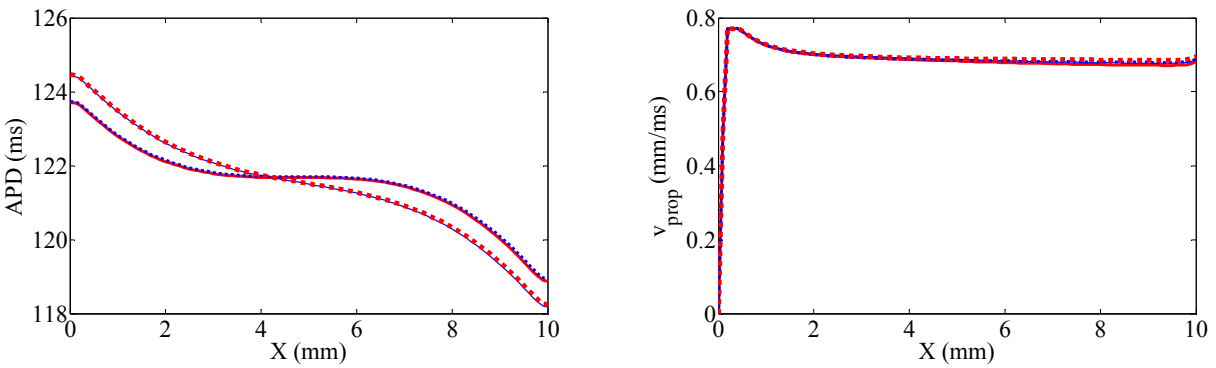

Figure 2. Spatial distributions of electrical variables shown in the reference configuration in case of isometric simulations with a preload equal to $4 \mathrm{kPa}$ and involving the healthy fiber: activation time $A T$ (A), repolarization time $R T(\mathrm{~B})$, action potential duration $A P D$ (C) and propagation velocity $v_{\text {prop }}$ (D). The different curves belong to a simulation with the conduction feedback alone (red, continuous) or together with the convection feedback (blue, dotted), the mechanoelectric feedback (blue, continuous) or both of them (red, dotted).

In the remainder of this work, we always include the effects of all feedbacks. Therefore, to better compare the electrical responses of healthy and hypertrophic fibers, we first collect in panels A and B of Figure 4 the spatial 

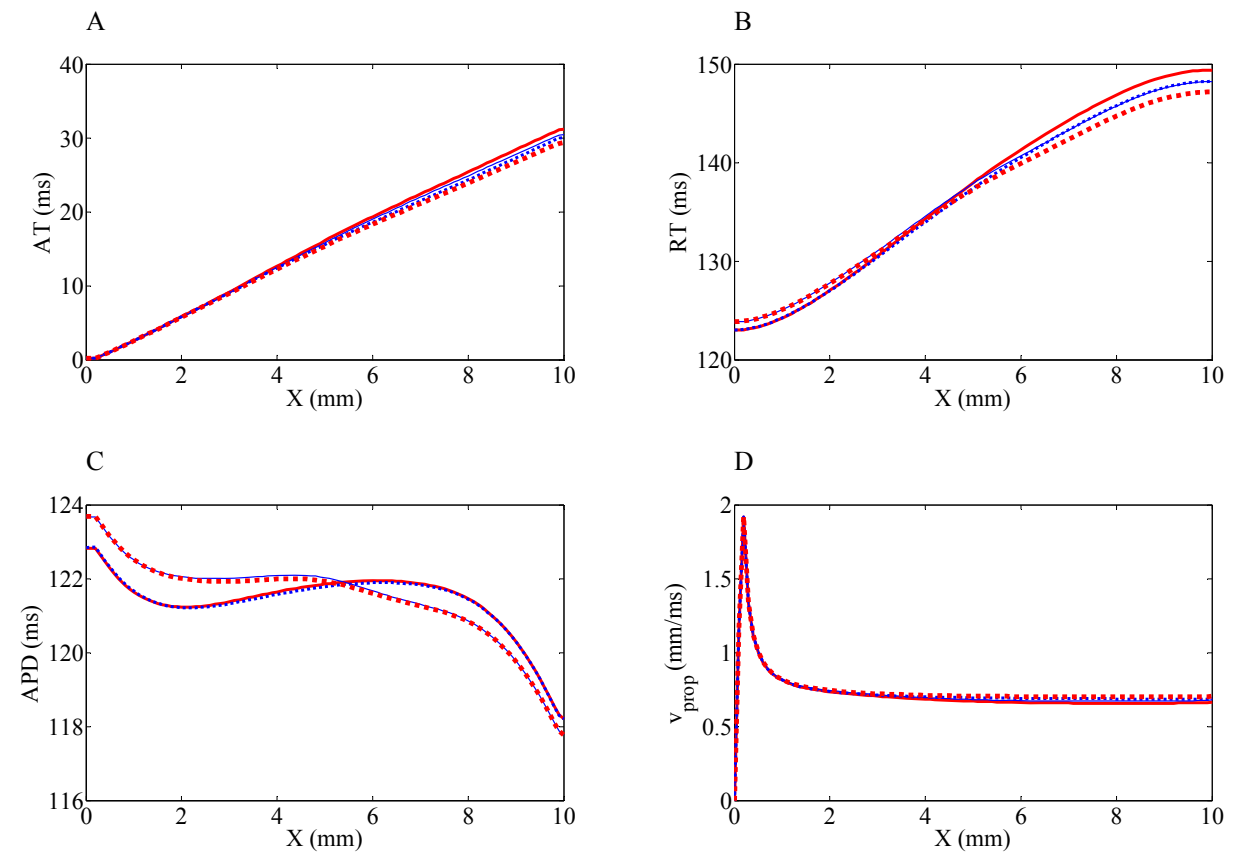

Figure 3. Spatial distributions of electrical variables shown in the reference configuration in case of isometric simulations with a preload equal to $4 k P a$ and involving the hypertrophic fiber. Same format as in Figure 2.

distributions of $A P D$ and $v_{\text {prop }}$ for the COND+CONV+SAC case. From panel $\mathrm{A}$, it turns out that the decreasing trend of $A P D$ for the hypertrophic fiber is less linear than the one for the healthy fiber, but the overall dispersion is not significantly affected. From panel B, hypertrophy alters $v_{\text {prop }}$ only close to the left end, where the stimulus is applied. Moreover, panels $\mathrm{C}$ and $\mathrm{D}$ display the time evolutions of the transmembrane potential $\mathrm{V}$ in those nodes where the maximum and minimum values for the growth parameter $\theta_{f}$ are detected compared with the corresponding time evolutions of the healthy case. Similarly, panels A and B of Figure 6 show the same evolutions for the hypertrophic fiber (with different local values for $\theta_{f}$ ) compared with the ones for the case with all local $\theta_{f}$ values equal to the mean of their distribution along the fiber (panel B of Figure 1). No significant differences are found from both figures again.

\subsection{The mechanical response}

The mechanical responses of healthy and hypertrophic fibers are studied by means of isometric, afterloaded isotonic and quick-release tests. 

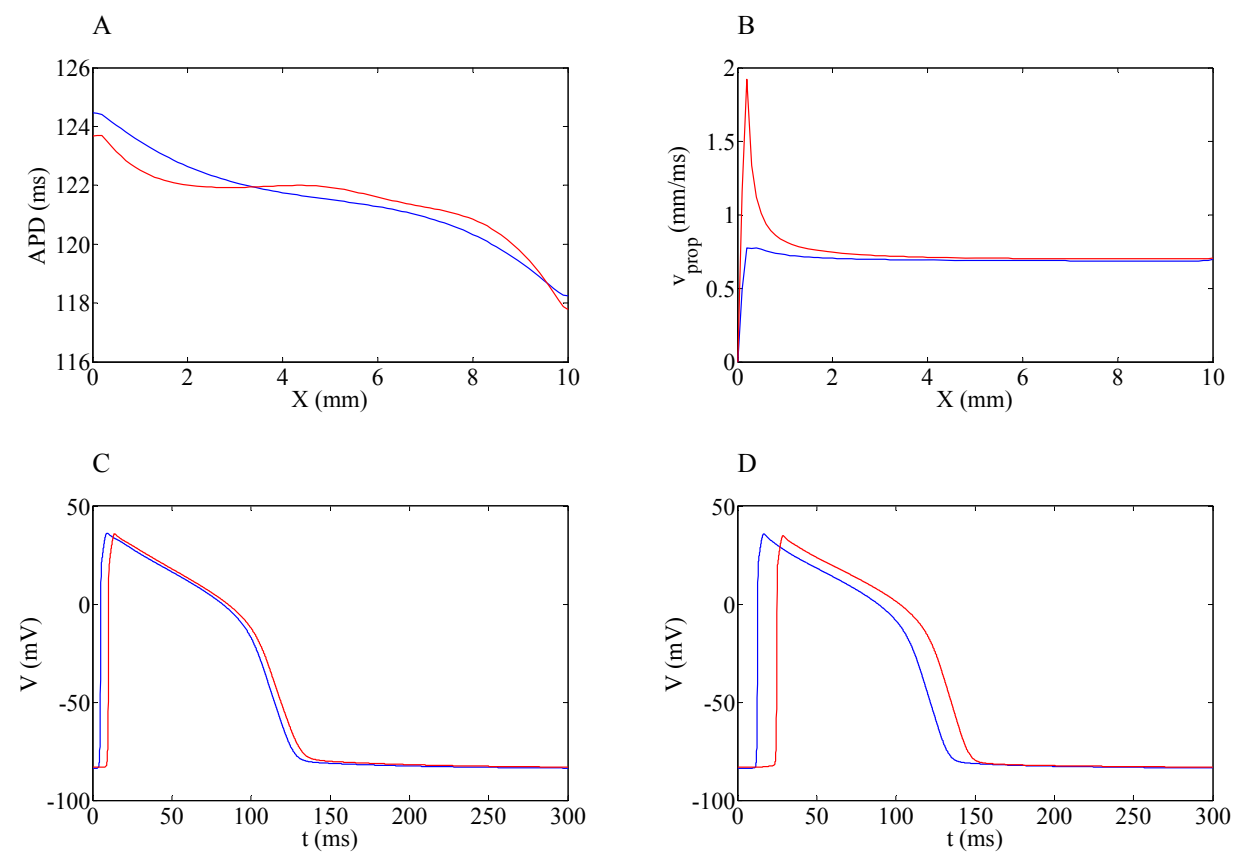

Figure 4. Electrical responses of the healthy (blue) and hypertrophic (red) fibers in case of isometric simulations with a preload equal to $4 k P a$ and all mechanical feedbacks. A-B) spatial distributions of the action potential duration APD (A) and propagation velocity $v_{\text {prop }}(\mathrm{B})$ shown in the reference configuration. C-D) time evolutions of the transmembrane potential $V$ in the nodes with the maximum (C) and minimum (D) values for $\theta_{f}$ in case of the hypertrophic fiber compared with the corresponding evolutions of the healthy case.

As regards the isometric tests, panel A of Figure 5 shows the time evolutions of the tension $T$ developed by the healthy and hypertrophic fibers at the left end after their electrical stimulation when the preload is varied $(0,1,2,3$ or $4 \mathrm{kPa})$. The peak values of curves get higher and higher as the preload increases, but they decrease with hypertrophy. Panel B reports the length-tension relationships, where the tensions before and after the electrical stimulation are represented as function of the increments in muscle length $\Delta L$, caused by the applied preloads. Again, the peak value for the tension developed after the electrical stimulation $T_{\max }$ decreases owing to hypertrophy. This result suggests that, in a three-dimensional environment, eccentric hypertrophic ventricles may develop a lower pressure during their isovolumic systole. Moreover, if the same preload is taken into account in panel B, it appears that the hypertrophic fiber gets more stretched than the healthy one during the preloading phase according to panel $\mathrm{F}$ of Fig- 


\section{F. Del Bianco et al.}

ure 1, where the values of the elastic stretch $\lambda^{e}$ are generally higher for the former fiber. This phenomenon is peculiar to cardiac fibers that are more likely to cause ventricular dilation during the diastolic filling, thus leading to a higher end-diastolic volume. Interestingly, both previous results are obtained without altering the mechanical parameters belonging to the strain energy function (5). Furthermore, for the hypertrophic fiber, the previous decrease in $T_{\max }$, which includes both active and passive components, occurs while the active tension $T_{a}$ in panel $\mathrm{E}$ of Figure 1 increases due to the higher values of $\lambda^{e}$. Then, similarly to $A P D$, panels $\mathrm{C}$ and $\mathrm{D}$ display the time evolutions of $\lambda^{e}$ where the maximum and minimum values for the growth parameter $\theta_{f}$ are found, comparing them with the healthy case. Moreover, panels $\mathrm{C}$ and $\mathrm{D}$ of Figure 6 display the same evolutions for the hypertrophic fiber (with different local values for $\theta_{f}$ ) compared with the ones for the case with a constant $\theta_{f}$ equal to the mean value along the fiber. From Figures 5 and 6, it appears that, where $\theta_{f}$ is maximum (panels $\mathrm{C})$, contraction is depressed. Conversely, where $\theta_{f}$ is minimum (panels D), contraction is enhanced. This proves that the more growth gets heterogeneous along the fiber the more the resulting mechanical response during the isovolumic systole of the cardiac cycle gets heterogeneous too. As far as the afterloaded isotonic and quick-release tests are concerned, panel A of Figure 7 illustrates an example of the time evolutions of the tension $T$ developed by the two fibers during the afterloaded isotonic contractions with a preload equal to $4 k P a$, whereas panel $\mathrm{B}$ depicts the corresponding time evolutions of the fiber length $L$. Panels A and B of Figure 8, instead, show the corresponding time evolutions during the quick-release contractions under the same preload. By considering all preloads from 0 to $4 \mathrm{kPa}$, tension-velocity relationships are derived from both afterloaded isotonic and quick-release tests (panels $\mathrm{C}$ ). The afterloads on the abscissa correspond to the developed tensions during the isotonic phase, whereas the contraction velocities $v_{\text {contr }}$ on the ordinate (taken positive) are computed from the constant slopes of the length curves in time as soon as the two fibers start to contract isotonically $[10,14]$. In particular, when the afterload is zero, the initial reduction of the fiber length up to the rest value within the first $m s$ in panel B of Figure 7 must be neglected for the measurement of the isotonic contraction velocity. In our simulations, the values for the velocity at a zero afterload are the same during the afterloaded isotonic tests, whilst they change during the quick-release ones. This different result may come out from the nature of the test itself. On one hand, the afterloaded isotonic test is the protocol that mimics best the in vivo transition from the isometric contraction to the isotonic one. On the other hand, instead, the quick-release test is the one that most approaches the in vitro experiments 
A



$\mathrm{C}$

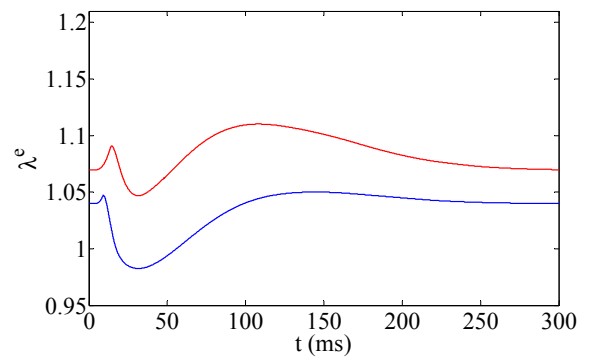

B

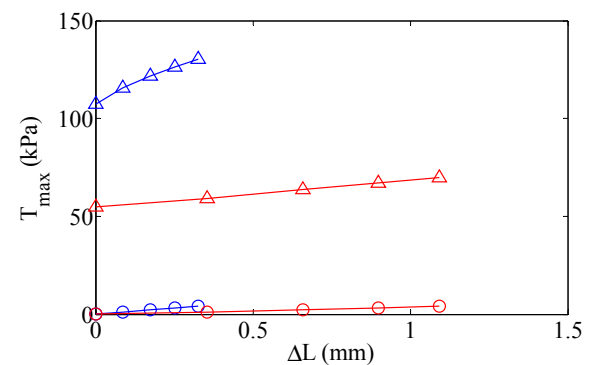

$\mathrm{D}$

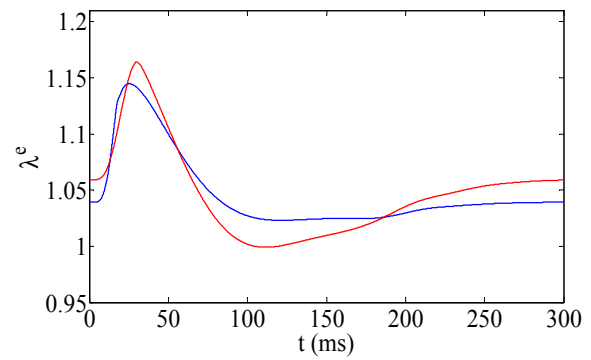

Figure 5. Mechanical responses of the healthy (blue) and hypertrophic (red) fibers in case of isometric simulations. A) time evolutions of the tension $T$ developed by the two fibers after their electrical stimulation for increasing preloads $(0,1,2,3$ or $4 k P a)$. B) corresponding length-tension relationships. $\Delta L$ on the abscissa is the increment in fiber length from the initial value according to the type of fiber, whereas $T_{\max }$ on the ordinate is the maximum value for the five tensions developed before (circles) and after (triangles) the electrical stimulation. C-D) time evolutions of the elastic stretch $\lambda^{e}$ in the nodes with the maximum (C) and minimum (D) values for $\theta_{f}$ in case of the hypertrophic fiber compared with the corresponding evolutions of the healthy case (preload equal to $4 \mathrm{kPa}$ ).

run on tetanized skeletal muscles, which cardiac fibers are often compared with; actually, although the heart cannot be tetanized, researchers try to reach an equally-activated muscle state by making constant the time during contraction at which the isotonic contraction velocity is measured. This discrepancy may lead to tension-velocity relationships that are not similar to hyperbolas (especially for small afterloads as in this work) when they are derived from afterloaded isotonic tests, whilst they could be entirely fitted to hyperbolas in case of quick-release tests. Nevertheless, from both tests, higher values of contraction velocity are reached by the hypertrophic fiber at smaller afterloads (and at a zero one accordingly), whereas lower values are found at bigger afterloads. The same trend characterizes the maximum shortenings $\Delta L_{\max }$ (that are nearly the same for both tests too) during 
F. Del Bianco et al.
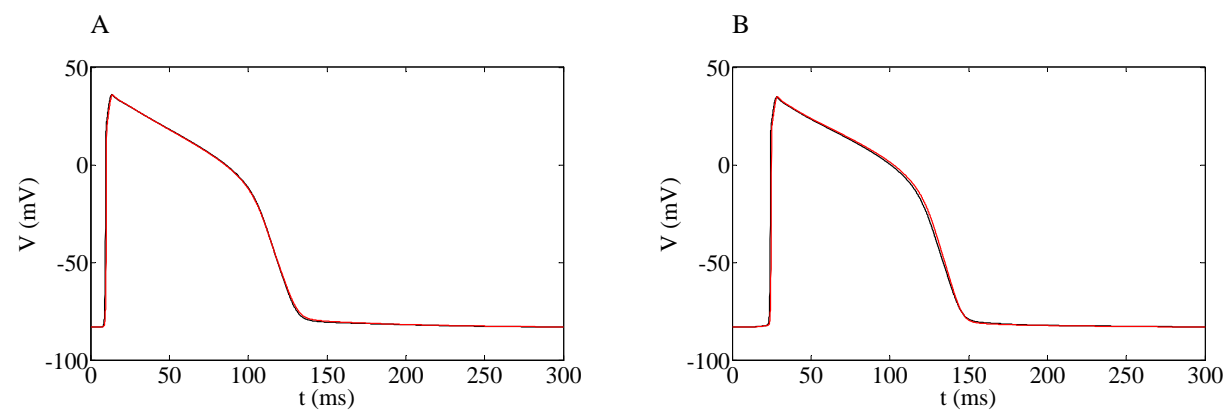

C

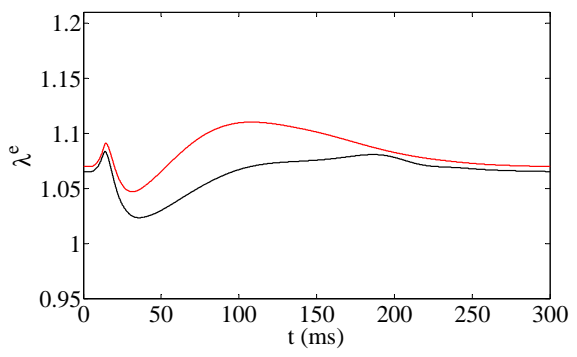

$\mathrm{D}$

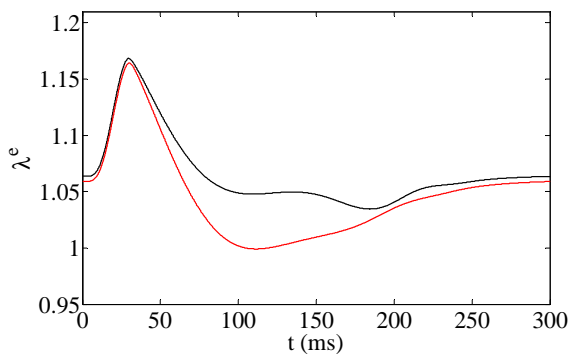

Figure 6. Time evolutions of electrical and mechanical variables in the nodes with the maximum (A-C) and minimum (B-D) values for $\theta_{f}$ in case of the hypertrophic fiber with an inhomogeneous growth (red) compared with the ones in the same nodes for the fiber with a homogeneous growth (black), i.e. with all local $\theta_{f}$ values equal to their mean along the fiber: A-B) transmembrane potential $V$; C-D) elastic stretch $\lambda^{e}$. These results belong to isometric simulations with a preload equal to $4 \mathrm{kPa}$ and all mechanical feedbacks.

the isotonic phase under each applied afterload in panels D of Figures 7 and 8. The two previous results point out that, in case of small afterloads (for example, when the aortic resistance to blood flow is physiological), eccentric hypertrophy may determine greater volume variations for a threedimensional ventricle during the systolic blood efflux phase of the cardiac cycle.

\section{Conclusions}

In this work, a one-dimensional strongly-coupled model has been developed to simulate the electromechanical activity of a ventricular fiber under healthy conditions or affected by eccentric hypertrophy while it contracts according to different protocols. Hypertrophy has been implemented both in the electrophysiological model and in the mechanical one to better analyze the full electromechanical response of contracting fibers. First, the 

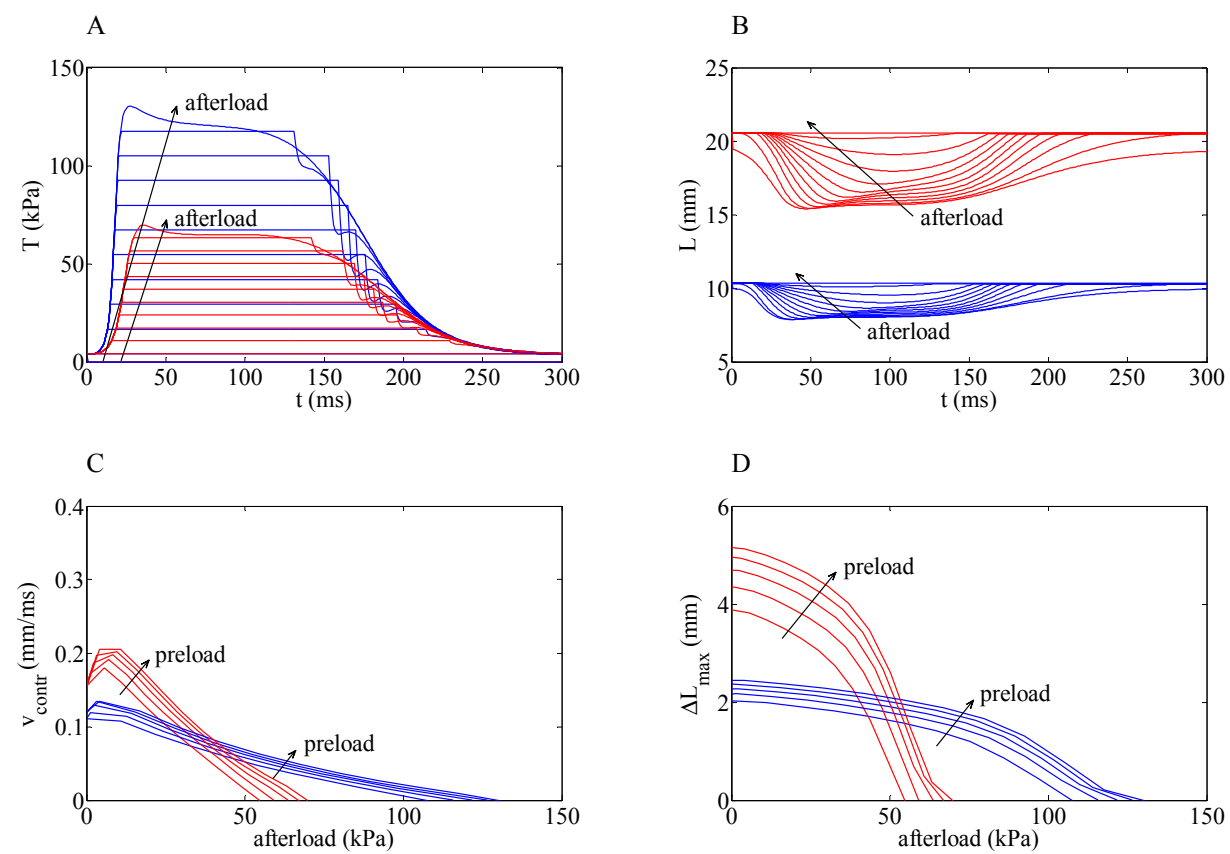

Figure 7. Mechanical responses of the healthy (blue) and hypertrophic (red) fibers in case of afterloaded isotonic simulations. A) time evolutions of the tension $T$ developed by the two fibers after their electrical stimulation with a preload equal to $4 \mathrm{kPa}$ and afterloads ranging from the minimum to the maximum value of $T$. B) corresponding time evolutions of the fiber length $L$. C) tension-velocity curves for increasing preloads $(0,1$, 2,3 or $4 \mathrm{kPa}$ ). The afterloads on the abscissa are equal to the tensions developed by the two fibers during their isotonic phase. The isotonic contraction velocities $v_{\text {contr }}$ are taken positive. D) corresponding maximum shortenings $\Delta L_{\max }$.

effects of the geometric feedbacks and of the mechanoelectric one on the electrical response of both healthy and hypertrophic fibers have been investigated. Then, by including all feedbacks, the electrical and mechanical responses of such fibers have been compared too.

It has turned out that neglecting one or more feedbacks does not significantly alter the behavior of both fibers. The effect of growth on electrophysiology is limited, suggesting that eccentric hypertrophy does not increment the risk of inducing arrhythmogenic phenomena when it is just a compensatory process to overload and a single fiber is taken into account. Future simulations should compare the results obtained in this work on fibers with the corresponding ones on three-dimensional dilated ventricles in order to test the existence of possible differences. Moreover, they may include the effects of eccentric growth at a molecular level through one or more genetic 
F. Del Bianco et al.
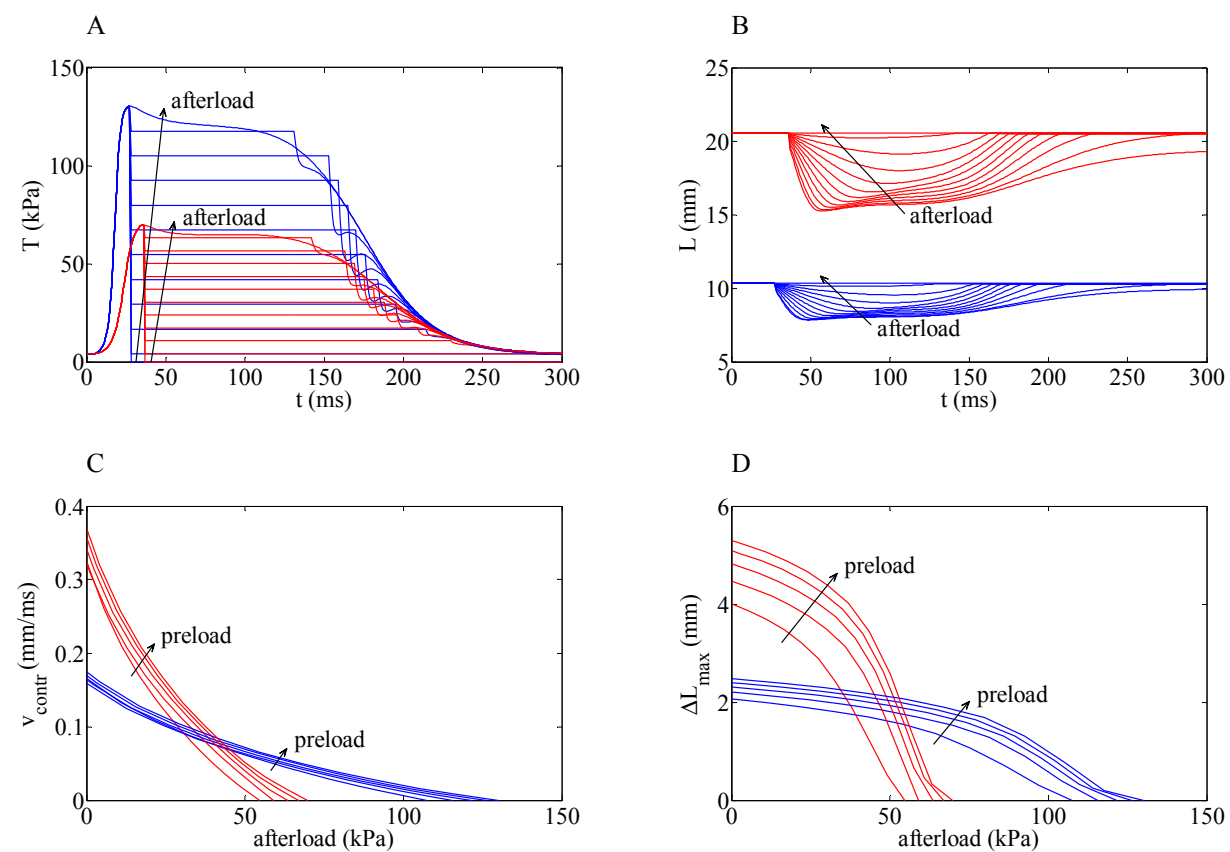

Figure 8. Mechanical responses of the healthy (blue) and hypertrophic (red) fibers in case of quick-release simulations. Same format as in Figure 7.

defects (characterizing hereditary hypertrophy), which could not preserve the shape of the action potential due to modifications involving the total number of membrane channels and gap junctions. In this way, a stronger and more pathological electrical remodeling could be triggered similarly to the one usually reported by the clinical literature.

Nevertheless, the effect of growth on fiber mechanics already suggests a pressure-volume loop for eccentric hypertrophic ventricles that enlarges over volumes and is more likely to shrink over pressures.

\section{REFERENCES}

1. C. Mihl, W. R. Dassen, and H. Kuipers, Cardiac remodelling: concentric versus eccentric hypertrophy in strength and endurance athletes, Neth. Heart J., vol. 16, pp. 129-133, 2008.

2. V. Kumar, A. K. Abbas, and N. Fausto, Robbins and Cotran Pathologic Basis of Disease. Philadelphia: Elsevier Saunders, 2005.

3. J. Ross Jr., Dilated cardiomyopathy: concepts derived from gene deficient and transgenic animal models, Circ. J., vol. 66, pp. 219-224, 2002. 


\section{Electromechanical modeling of an eccentric hypertrophic fiber}

4. E. Berberoglu, H. O. Solmaz, and S. Goktepe, Computational modeling of coupled cardiac electromechanics incorporating cardiac dysfunctions, Eur. J. Mech. - A/Solids, vol. 48, pp. 60-73, 2014.

5. S. Goktepe, O. J. Abilez, K. K. Parker, and E. Kuhl, A multiscale model for eccentric and concentric cardiac growth through sarcomerogenesis, J. Theor. Biol., vol. 265, pp. 433-442, 2010.

6. R. C. Kerckhoffs, J. Omens, and A. D. McCulloch, A single strain-based growth law predicts concentric and eccentric cardiac growth during pressure and volume overload, Mech. Res. Commun., vol. 42, pp. 40-50, 2012.

7. L. C. Lee, J. Sundnes, M. Genet, J. F. Wenk, and S. T. Wall, An integrated electromechanical-growth heart model for simulating cardiac therapies, Biomech. Model. Mechanobiol., vol. 15, pp. 791-803, 2016.

8. L. B. Katsnelson, N. A. Vikulova, A. G. Kursanov, O. E. Solovyova, and V. S. Markhasin, Electro-mechanical coupling in a one-dimensional model of heart muscle fiber, Russian J. Numer. Anal. Math. Model., vol. 29, pp. 1-13, 2014.

9. N. H. Kuijpers, H. M. ten Eikelder, P. H. Bovendeerd, S. Verheule, T. Arts, and P. A. Hilbers, Mechanoelectric feedback leads to conduction slowing and block in acutely dilated atria: a modeling study of cardiac electromechanics, Am. J. Physiol. Heart Circ. Physiol., vol. 292, pp. H2832-H2853, 2007.

10. M. I. Noble, T. E. Bowen, and L. L. Hefner, Force-velocity relationship of cat cardiac muscle, studied by isotonic and quick-release techniques, Circ. Res., vol. 24, pp. 821-833, 1969.

11. N. A. Vikulova, L. B. Katsnelson, A. G. Kursanov, O. Solovyova, and V. S. Markhasin, Mechano-electric feedback in one-dimensional model of myocardium, J. Math. Biol., vol. 73, pp. 335-366, 2016.

12. S. A. Niederer and N. P. Smith, An improved numerical method for strong coupling of excitation and contraction models in the heart, Prog. Biophys. Mol. Biol., vol. 96, pp. 90-111, 2008.

13. J. P. Whiteley, M. J. Bishop, and D. J. Gavaghan, Soft tissue modelling of cardiac fibres for use in coupled mechano-electric simulations, Bull. Math. Biol., vol. 69, pp. 2199-2225, 2007.

14. E. H. Sonnenblick, Force-velocity relations in mammalian heart muscle, Am. J. Physiol., vol. 202, pp. 931-939, 1962.

15. P. Colli Franzone, L. F. Pavarino, and S. Scacchi, Biolectrical effects of mechanical feedbacks in a strongly coupled cardiac electro-mechanical model, Math. Mod. Meth. Appl. S., vol. 26, pp. 27-57, 2016.

16. P. Kohl, F. Sachs, and M. R. Franz, Cardiac Mechano-electric Coupling E Arrhythmias. New York: Oxford University Press, 2011. 


\section{F. Del Bianco et al.}

17. M. P. Nash and A. V. Panfilov, Electromechanical model of excitable tissue to study reentrant cardiac arrhythmias, Prog. Biophys. Mol. Biol., vol. 85, pp. 501-522, 2004.

18. A. V. Panfilov, R. H. Keldermann, and M. P. Nash, Self-organized pacemakers in a coupled reaction-diffusion-mechanics system, Phys. Rev. Lett., vol. 95, p. 258104, 2005.

19. G. A. Holzapfel, Nonlinear Solid Mechanics: a Continuum Approach for Engineering. Chichester: John Wiley \& Sons, 2000.

20. E. K. Rodriguez, A. Hoger, and A. D. McCulloch, Stress-dependent finite growth in soft elastic tissues, J. Biomech., vol. 27, pp. 455-467, 1994.

21. D. Ambrosi, G. A. Ateshian, E. M. Arruda, S. C. Cowin, J. Dumais, A. Goriely, G. A. Holzapfel, J. D. Humphrey, R. Kemkemer, E. Kuhl, J. E. Olberding, L. A. Taber, and K. Garikipati, Perspectives on biological growth and remodeling, J. Mech. Phys. Solids, vol. 59, pp. 863-883, 2011.

22. P. Pathmanathan and J. P. Whiteley, A numerical method for cardiac mechanoelectric simulations, Ann. Biomed. Eng., vol. 37, pp. 860-873, 2009.

23. H. M. Wang, H. Gao, X. Y. Luo, C. Berry, B. E. Griffith, R. W. Ogden, and T. J. Wang, Structure-based finite strain modelling of the human left ventricle in diastole, Int. J. Numer. Method. Biomed. Eng., vol. 29, pp. 83-103, 2013.

24. M. K. Rausch, A. Dam, S. Gï $; \frac{1}{2}$ ktepe, O. J. Abilez, and E. Kuhl, Computational modeling of growth: systemic and pulmonary hypertension in the heart, Biomech. Model. Mechanobiol., vol. 10, pp. 799-811, 2011.

25. S. Land, S. A. Niederer, J. M. Aronsen, E. K. Espe, L. Zhang, W. E. Louch, I. Sjaastad, O. M. Sejersted, and N. P. Smith, An analysis of deformation-dependent electromechanical coupling in the mouse heart, J. Physiol., vol. 590, pp. 4553-4569, 2012.

26. G. M. Faber and Y. Rudy, Action potential and contractility changes in $\left[\mathrm{Na}^{+}\right]_{i}$ overloaded cardiac myocytes: a simulation study, Biophys. J., vol. 78, pp. 2392-2404, 2000.

27. P. Colli Franzone, L. F. Pavarino, and B. Taccardi, Simulating patterns of excitation, repolarization and action potential duration with cardiac bidomain and monodomain models, Math. Biosci., vol. 197, pp. 35-66, 2005.

28. P. Colli Franzone, L. F. Pavarino, and S. Scacchi, Mathematical Cardiac Electrophysiology. Cham: Springer, 2014.

29. C. S. Henriquez, Simulating the electrical behavior of cardiac tissue using the bidomain model, Crit. Rev. Biomed. Eng., vol. 21, pp. 1-77, 


\section{Electromechanical modeling of an eccentric hypertrophic fiber}

1993.

30. B. J. Roth, The electrical potential produced by a strand of cardiac muscle: a bidomain analysis, Ann. Biomed. Eng., vol. 16, pp. 609-637, 1988.

31. R. M. Shaw and Y. Rudy, Ionic mechanisms of propagation in cardiac tissue. Roles of the sodium and L-type calcium currents during reduced excitability and decreased gap junction coupling, Circ. Res., vol. 81, pp. 727-741, 1997.

32. S. A. Niederer and N. P. Smith, A mathematical model of the slow force response to stretch in rat ventricular myocytes, Biophys. J., vol. 92, pp. 4030-4044, 2007.

33. W. Kroon, T. Delhaas, T. Arts, and P. H. Bovendeerd, Computational modeling of volumetric tissue growth: application to the cardiac left ventricle, Biomech. Model. Mechanobiol., vol. 8, pp. 301-309, 2009.

34. S. Rush and H. Larsen, A practical algorithm for solving dynamic membrane equations, IEEE Trans. Biomed. Eng., vol. 25, pp. 389-392, 1978. 